\title{
Burning issues in the prevention of heart failure
}

\author{
JA Ker, ${ }^{1}$ K Outhoff ${ }^{2}$ \\ ${ }^{1}$ Department of Internal Medicine, School of Medicine, Faculty of Health Sciences, University of Pretoria, South Africa \\ ${ }^{2}$ Department of Pharmacology, School of Medicine, Faculty of Health Sciences, University of Pretoria, South Africa \\ Corresponding author, email: kim.outhoff@up.ac.za
}

Chronic heart failure is common, debilitating, and often the culmination of pervasive cardiovascular insults that systematically undermine the heart's circulatory capacity and invoke counterproductive neuro-hormonal compensatory changes. Prevention of chronic heart failure therefore requires minimising the impact of traditional cardiovascular risk factors with incisive treatment of hypertension and type II Diabetes Mellitus (T2DM) and prompt lifestyle interventions for smoking, lack of exercise, obesity and hypercholesterolemia. This review is narrative, with selected emphasis on major studies, rather than structured on a specific clinical question, and should be read as such.

Keywords: Chronic heart failure; prevention; cardio-protection; hypertension; type 2 Diabetes Mellitus

\section{Background}

About $2 \%$ of the adult population worldwide is affected by chronic heart failure and this figure rises steeply with increasing age, especially from age 50 , to about $10 \%$ in adults older than 75 years of age. ${ }^{1}$ The life-time risk of developing heart failure at age 40 years is about $20 \%$ for both men and women. ${ }^{2}$ Ageing populations and improved treatment of acute cardiovascular events are contributing to the predicted $25 \%$ increase in heart failure in the next 20 years. ${ }^{3}$ Patients who suffer from heart failure have a poor overall prognosis and have high rates of hospital admissions and medical care costs. These facts necessitate that emphasis be placed on the prevention of heart failure as an important and critical health care issue.

Heart failure has many various causes and complicated pathophysiological pathways, but essentially the greatest contributors to the risk of developing heart failure remain traditional cardiovascular risk factors which include hypertension, diabetes mellitus, elevated cholesterol, smoking, unhealthy lifestyle, obesity and lack of exercise. ${ }^{4}$ The development of heart failure generally proceeds in stages. Stage A: Risk factors are present but there are no symptoms or signs of heart failure. Stage B: There are still no symptoms or signs of heart failure but the heart has a structural abnormality such as left ventricular hypertrophy, previous myocardial infarction or a cardiac murmur and there could also be a functional cardiac abnormality such as asymptomatic reduction of the ejection fraction. Stage C: There are now symptoms and signs of heart failure. This is clinical heart failure. ${ }^{5}$ Once a patient has reached a specific stage, therapy cannot reverse the stage but can reduce the risk of progression.

\section{Identify and treat Stage B heart failure}

Preventative treatment with renin-angiotensin-aldosteronesystem (RAAS)-blockers is indicated in high-risk individuals without known ventricular dysfunction, in asymptomatic patients with known left ventricular systolic dysfunction, in people with recent myocardial infarction and left-ventricular dysfunction. Beta-blockers are also indicated post-myocardial infarction and left-ventricular dysfunction.

\section{Treatment of hypertension to prevent heart failure}

Several meta-analyses have assessed heart failure endpoints in blood pressure lowering studies. In a large meta-analysis of approximately 1 million people in 61 prospective observational studies published in 2002, it was shown that long-term average reductions of $20 \mathrm{mmHg}$ in usual systolic blood pressure halved the risk of heart failure mortality. ${ }^{6}$ The hazard ratio of 0.53 (95\% $\mathrm{Cl}: 0.48-0.59)$ in this study underscored the significant reduction in heart failure with a lower usual blood pressure. The absolute benefit of a lower blood pressure is likely to be greatest for those at a higher cardiovascular risk.

A 2014 meta-analysis of 68 randomized clinical trials involving 235885 participants showed that a reduction of $10 \mathrm{mmHg}$ systolic and $5 \mathrm{mmHg}$ diastolic blood pressure for 5 years was associated with a significant reduction of heart failure. The relative reduction in heart failure was 38\% (95\% Cl: $25-49 \%)$ with an absolute risk reduction of 19 (95\%Cl: $13-24)$ per 1000 patients over 5 years, with a number needed-to-treat of $53(95 \% \mathrm{Cl}$ : 49 79). ${ }^{7}$ The meta-analysis inclusion criteria were broad, as heart failure is not always an end-point in hypertension studies. In fact, of the 68 , only 38 trials on hypertension could be included in this particular analysis. The authors also remarked that the reductions of heart failure as well as stroke were greater and more significant than any other cardiovascular outcome in these trials.

In 2016, another meta-analysis of 123 studies and 613815 hypertensive patients showed that for every $10 \mathrm{mmHg}$ reduction of systolic blood pressure, the relative risk of heart failure was 
reduced by $28 \%$ (95\%Cl: $22-33 \%) .{ }^{8}$ Unfortunately, absolute risk reductions were not calculated.

Conversely, in a meta-analysis of 40 trials investigating blood pressure lowering in type 2 diabetes involving 100354 participants, the reduction of blood pressure did not lead to significant reductions in heart failure in diabetics. ${ }^{9}$ This unexpected result could possibly be attributed to the smaller sample of randomised trials that evaluated heart failure as an end-point. Meanwhile, a 2018 meta-analysis of 74 trials and 306 273 participants found it difficult to interpret the data on heart failure when outcomes based on baseline blood pressure values were assessed. ${ }^{10}$

\section{Targeting people at high cardiovascular risk}

Assessing cardiovascular risk is an important part of primary care, especially if early interventions are implemented successfully. Brain-type natriuretic peptide (BNP) screening of patients with cardiovascular risk factors and subsequent improved risk factor control and treatment with RAAS-inhibitors reduced the rate of asymptomatic left ventricular dysfunction or overt heart failure compared to normal (minimal) interventions. ${ }^{11}$ In patients with coronary artery disease without left ventricular dysfunction or heart failure, and in those patients with asymptomatic left ventricular dysfunction, regardless of aetiology, the use of RAASinhibitors reduce the risk of hospital admissions due to heart failure.

\section{Diabetes Mellitus type-2}

Type 2 Diabetes Mellitus (T2DM) is an important contributor to the development of heart failure. Usually, T2DM causes ischaemic heart disease, which leads to heart failure, but DM is also commonly associated with hypertension, and this combination is independently associated with an increased risk of heart failure. T2DM on its own is also associated with heart failure, a condition called diabetic cardiomyopathy. Interestingly, glycaemic control per se is not associated with a reduced risk of heart failure and some of the glitazones used as therapy may even lead to worsening heart failure.

Conversely, the sodium-glucose cotransporter-2 inhibitors (SGLT2 inhibitors) have been studied in T2DM and have consistently shown a reduction of hospitalization for heart failure. A recent meta-analysis showed that the risk of hospitalization for heart failure is reduced by a relative risk reduction of $23 \%$ (RR 0.77 ; $95 \% \mathrm{Cl}: 0.71-0.84 ; p<0.0001)$ with a similar benefit in patients with and without atherosclerotic cardiovascular disease and with and without a history of heart failure. ${ }^{12}$

\section{Role of statins}

Statins have not really been evaluated carefully for their effect on heart failure, and the available data are sparse. There is, however, a publication suggesting that the use of statins may reduce the risk of heart failure. ${ }^{13}$

\section{Lifestyle factors}

Adherence to a healthy lifestyle, such as smoking cessation, regular exercise, healthy weight, moderate alcohol intake and consumption of high fibre and fruit and vegetables, is associated with a reduction in the risk of developing heart failure..$^{14}$ Other studies have reported the same beneficial effects of a healthy lifestyle.

\section{Concluding summary}

1. Heart failure has a poor prognosis and can develop from virtually any cardiac condition.

2. Preventing heart failure is an important aspect of cardiovascular practice and the high-risk patient should be identified in clinical practice for preventative therapy.

3. Proper treatment of hypertension with adequate control of blood pressure is probably the most important and significant aspect of preventing the development of heart failure.

4. Identifying others at high risk of developing heart failure, such as those surviving an atherosclerotic cardiac event, and treating them appropriately, reduces the risk of heart failure.

5. Attention to improving lifestyle factors should always be part of the prevention of heart failure over a lifetime as there is proven benefit.

\section{Orcid}

K Outhoff https://orcid.org/0000-0002-0851-4802

\section{References}

6. Metra M, Teerlink JR. Heart failure. Lancet. 2017;390:1981-95.

7. Lloyd-Jones DM, Larson MG, Leip EP, et al. Life-time risk for developing congestive heart failure: the Framingham Heart Study. Circulation. 2002;106:3068-72.

8. Heidenreich PA, Albert NM, Allen LA, et al. Forecasting the impact of heart failure in the United States: a policy statement from the American Heart Association. Cir Heart Fail. 2013;6:606-19.

9. Nambi V, Deswal A, Ballantyne CM. Prevention of "failure": Is it a failure of prevention? Circulation. 2018;137:106-8.

10. Hunt SA, Abraham WT, Chin MH, et al. ACC/AHA 20015 guideline update for the diagnosis and management of chronic heart failure in the adult: a report of the American College of Cardiology/American Heart Association Task Force on Practice Guidelines. Circulation. 2005;112:e154-235.

11. Lewington S, Clarke R, Qizilbasin N, et al for the Prospective Studies Collaboration. Age-specific relevance of usual blood pressure to vascular mortality: a meta-analysis of individual data from one million adults in 61 prospective studies. Lancet. 2001;360:1903-13.

12. Thomopoulos C, Parati G, Zanchetti A. Effects of blood pressure lowering on outcome incidence in hypertension.1. Overview, meta-analyses and meta-regression analyses of randomized trials. J Hypertension. 2014;32:2285-95.

13. Ettehad D, Emdin CA, Kiran A, et al. Blood pressure lowering for prevention of cardiovascular disease and death: a systematic review and meta-analysis. Lancet. 2016;387:957-67.

14. Emdin CA, Rahimi K, Neal B, et al. Blood pressure lowering in type 2 diabetes: a systematic review and meta-analysis. JAMA. 2015;313:603-15.

15. Brunström M, Carlberg Bo. Association of blood pressure lowering with mortality and cardiovascular disease across blood pressure levels: a systematic review and meta-analysis. JAMA Intern Med. 2018;178:28-36.

16. Ledwige $M$, Gallagher J, Conlon $C$, et al. Natriuretic peptide-based screening and collaborative care for heart failure: The STOP-HF randomised trial. JAMA 2013;310:66-74.

17. Zelniker TA, Wiviott SD, Raz l, et al. SGLT2 inhibitors for primary and secondary prevention of cardiovascular and renal outcomes in type 2 diabetes: a systematic review and meta-analysis of cardiovascular outcome trials. Lancet. 2019:393:31-9.

18. Preiss D, Campbell RT, Murray HM, et al. The effect of statin therapy on heart failure events: a collaborative meta-analysis of unpublished data from major randomised trials. Eur Heart J. 2015;36:1536-46.

19. Djousse L, Driver JA, Gaziano JM. Relation between modifiable lifestyle factors and lifetime risk of heart failure. JAMA. 2009;302:394-400. 